\title{
Further Results on Dynamic Additive Hazard Rate Model
}

\author{
Zhengcheng Zhang ${ }^{1}$ and Limin Zhang ${ }^{2}$ \\ ${ }^{1}$ School of Mathematics and Physics, Lanzhou Jiaotong University, P.O. Box 405, Anning West Road, Anning District, \\ Lanzhou, Gansu 730070, China \\ ${ }^{2}$ Department of Applied Mathematics, College of Basic Science and Information Engineering, Yunnan Agricultural University, \\ Kunming, Yunnan 650201, China \\ Correspondence should be addressed to Zhengcheng Zhang; zhzhcheng004@163.com
}

Received 15 April 2014; Revised 22 June 2014; Accepted 7 July 2014; Published 21 July 2014

Academic Editor: Wuquan Li

Copyright $\odot 2014$ Z. Zhang and L. Zhang. This is an open access article distributed under the Creative Commons Attribution License, which permits unrestricted use, distribution, and reproduction in any medium, provided the original work is properly cited.

In the past, the proportional and additive hazard rate models have been investigated in the works. Nanda and Das (2011) introduced and studied the dynamic proportional (reversed) hazard rate model. In this paper we study the dynamic additive hazard rate model, and investigate its aging properties for different aging classes. The closure of the model under some stochastic orders has also been investigated. Some examples are also given to illustrate different aging properties and stochastic comparisons of the model.

\section{Introduction}

It is common practice in statistical analysis that covariates are often introduced to account for factors that increase the heterogeneity of a population. When the effect of a factor under study has a multiplicative (or additive) effect on the baseline hazard function, we have a proportional (or an additive) hazard model. The latter category of model is preferred in any situation. For example, in tumorigenicity cases, where the dose effect on tumor risk is of interest, the excess risk becomes an important factor. Clinical trials that seek the effectiveness of treatments often experience lag times of treatment effectiveness after which treatment procedures will be in full effect.

In reliability and survival analysis, devices or systems always operate in a changing environment. The conditions under which systems operate can be harsher or gentler in modeling lifetime of the devices or systems. The most known Cox [1] model is that the changing conditions are assumed to act multiplicatively on the baseline hazard rate. This model has been widely used in many experiments where the time to systems' failure depends on a group of covariates, which may be regarded as different treatments, operating conditions, heterogeneous environments, and so forth. P. L. Gupta and R. C. Gupta [2] studied the relation between the conditional and unconditional failure rates in mixtures when the distributions in the mixture follow the proportional hazard rate. For further research, one may see Cox and Oakes [3], Kumar and Westberg [4], Dupuy [5], Lau [6], Zhao and Zhou [7], X. Li and $\mathrm{Z}$. Li [8], and $\mathrm{Yu}[9]$.

R. C. Gupta and R. D. Gupta [10] proposed and studied the proportional reversed hazard model to analyze failure time data. For more details on this model, see Gupta and $\mathrm{Wu}$ [11], X. Li and Z. Li [12], and so forth.

Recently, Nanda and Das [13] introduced the dynamic proportional hazard rate (DPHR) model and the dynamic proportional reversed hazard rate (DPRHR) model and studied their properties for different aging classes. The closure of the models under different stochastic orders has also been studied.

Aranda-Ordaz [14] first dealt with an additive hazard model

$$
h(t \mid Z(s), s \leq t)=\beta^{\prime} z(t)+h_{0}(t), \quad \text { for } t \geq 0,
$$

where $h_{0}(t)$ is a baseline hazard rate and a time-dependent covariate vector $Z$, representing the changes in the operating conditions, and $\beta$ is a vector of parameters. For more details, one may see Cox and Oakes [3], Thomas [15], Breslow and Day [16], Finkelstein and Esaulova [17], Lim and Zhang [18], and so forth. 
Assume that $X$ and $Y$ are the lifetimes of two systems with corresponding hazard rate functions $h_{X}(t)$ and $h_{Y}(t)$ for $t \geq 0$. Let $c(t)=\beta^{\prime} z(t)$; the model (with time-dependent covariates) in (1) would reduce to the form

$$
h_{Y}(t)=c(t)+h_{X}(t), \quad \forall t \geq 0
$$

which is named as dynamic additive hazard rate (DAHR) model.

Sometimes the hazard rate functions of $X$ and $Y$ may not be additive over the whole interval $[0, \infty)$, but they may be additive differently from different intervals. Specifically, they may be related as

$$
h_{Y}(t)=c_{i}+h_{X}(t), \quad t_{i-1} \leq t \leq t_{i}
$$

for $i=1,2, \ldots$, and $t_{0}=0$, where $c_{i}(i=1,2, \ldots)$ are some constants. When the intervals $\left[t_{i-1}, t_{i}\right)(i=1,2, \ldots)$ become smaller and smaller, a model as in (2) will be naturally obtained.

In order to guarantee that $h_{Y}(t)$ is a hazard rate function of a nonnegative random variable $Y$, the following lemma is given.

Lemma 1. Assume that $c(t)$ and $h_{X}(t)$ are defined before. Then, for $t \geq 0, h_{Y}(t)=c(t)+h_{X}(t)$ is a hazard rate function if and only if the following conditions hold:

(i) $c(t)+h_{X}(t) \geq 0$, for all $t \geq 0$;

(ii) $\int_{0}^{\infty}\left(c(t)+h_{X}(t)\right) d t=\infty$;

(iii) if $\int_{0}^{t_{0}} h_{X}(t) d t=\infty$, then

$$
\int_{0}^{t_{0}}\left(c(t)+h_{X}(t)\right) d t=\infty
$$

for some $t_{0}<\infty$.

In Section 2 of the paper, we discuss some aging properties of the DAHR model. In Section 3, the closure of DAHR model under different stochastic orderings is studied. Some examples are given to illustrate the results concerned in Sections 2 and 3.

Throughout the paper, assume that all random variables under consideration have 0 as the common left end point of their supports, and the terms increasing and decreasing stand for monotone nondecreasing and monotone nonincreasing, respectively.

\section{Aging Properties of DAHR Model}

At first we introduce some concepts of aging notions that will be useful in the section. Recall that a random variable $X$ is said to be (a) increasing in failure rate (IFR) [decreasing in failure rate (DFR)] if $h_{X}(t)$ is increasing [decreasing] in $t \geq 0$; (b) increasing in failure rate in average (IFRA) [decreasing in failure rate in average (DFRA)] if $\int_{0}^{t} h_{X}(u) d u / t$ is increasing [decreasing] in $t \geq 0$; (c) new better than used (NBU) [new worse than used (NWU)] if $\bar{F}(x+t) \leq[\geq] \bar{F}(t) \bar{F}(x)$, for all $t, x \geq 0$; (d) new better than used in failure rate (NBUFR) [new worse than used in failure rate (NWUFR)] if $h_{X}(t) \geq(\leq$ $h_{X}(0)$, for all $t \geq 0$; (e) new better than used in failure rate average (NBAFR) [new worse than used in failure rate average (NWAFR) $]$ if $\int_{0}^{t} h_{X}(u) d u / t \geq[\leq] h_{X}(0)$, for all $t \geq 0$. For more discussions on properties of aging notions, readers may refer to Barlow and Proschan [19], Müller and Styan [20], and so forth.

In the following we give some aging closure properties between the random variables $X$ and $Y$ under some conditions of $c(t)$. Some results are obvious and hence their proofs are omitted.

Proposition 2. If the random variable $X$ is IFR (DFR) and, for $t \geq 0, c(t)$ is increasing (decreasing), then the random variable $Y$ is IFR (DFR).

In the following, we give two examples related to this proposition. Example 3 is an application of the proposition. Example 4 indicates that the condition of $c(t)$ is sufficient but not a necessary one.

Example 3. Let $X$ be a random variable having Weibull distribution with hazard rate function $h_{X}(t)=2 t, t \geq 0$. Take $c(t)=t$ for $t \geq 0$. It is obvious that $c(t)$ satisfies all the conditions of Lemma 1. Obviously, if $X$ is IFR and $c(t)$ is increasing in $t$, hence $Y$ is IFR.

Example 4. Let $X$ be a random variable having Weibull distribution with hazard rate function $h_{X}(t)=2 t, t \geq 0$. Let $c(t)=\left(2+t^{2}\right) /(1+t)$ for $t \geq 0$. It can be verified that $h_{X}(t)+c(t)$ is increasing in $t \geq 0$, and hence $Y$ is IFR. However, $c(t)$ is decreasing in $t \in[0, \sqrt{3}-1)$ but increasing in $t \in[\sqrt{3}-1,+\infty)$.

Proposition 5. If the random variable $X$ is IFRA (DFRA) and $c(t)$ is increasing (decreasing) in $t \geq 0$, then the random variable $Y$ is IFRA (DFRA).

Proof. For $t \geq 0$, let

$$
q(t)=\frac{\int_{0}^{t} h_{Y}(x) d x}{t}=\frac{\int_{0}^{t}\left(c(x)+h_{X}(x)\right) d x}{t} .
$$

Note that $X$ is IFRA (DFRA) and $c(t)$ is increasing (decreasing) implying that

$$
\begin{aligned}
q^{\prime}(t) & =\frac{c(t)+h_{X}(t)}{t}-\frac{\int_{0}^{t}\left(c(x)+h_{X}(x)\right) d x}{t^{2}} \\
& =\frac{\int_{0}^{t}(c(t)-c(x)) d x}{t^{2}}+\frac{t h_{X}(t)-\int_{0}^{t} h_{X}(x) d x}{t^{2}} \\
& \geq 0(\leq 0) .
\end{aligned}
$$

Hence the desired result follows directly.

Example 3 can be regarded as an application of the above proposition. Example 6 below indicates that the condition of 
$c(t)$ is sufficient but not a necessary one for the monotone property of $Y$.

Example 6. Let $X$ be a random variable having Weibull distribution with hazard rate function $h_{X}(t)=2 t, t \geq 0$. Take $c(t)=-t$ for $t \geq 0$. It is obvious that $c(t)$ satisfies all the conditions of Lemma 1. Obviously, $X$ is IFRA and $Y$ is IFRA. However, $c(t)$ is decreasing in $t \geq 0$.

Proposition 7. If the random variable $X$ is $N B U$ (NWU) and $c(t)$ is increasing (decreasing) in $t \geq 0$, then the random variable $Y$ is NBU (NWU).

Proof. We only give the proof for the case of NBU. In order to prove that $Y$ is NBU, it is sufficient to prove that, for all $t \geq 0$ and $x \geq 0$,

$$
\begin{aligned}
e^{-\int_{0}^{x+t}\left(c(u)+h_{X}(u)\right) d u} \leq & e^{-\int_{0}^{x}\left(c(u)+h_{X}(u)\right) d u} \\
& \times e^{-\int_{0}^{t}\left(c(u)+h_{X}(u)\right) d u} .
\end{aligned}
$$

It is equivalent to

$$
e^{-\int_{t}^{x+t}\left(c(u)+h_{X}(u)\right) d u} \leq e^{-\int_{0}^{x}\left(c(u)+h_{X}(u)\right) d u} .
$$

That is,

$$
e^{-\int_{0}^{x}\left(c(u+t)+h_{X}(u+t)\right) d u} \leq e^{-\int_{0}^{x}\left(c(u)+h_{X}(u)\right) d u} .
$$

Note that $X$ is $\mathrm{NBU}$ which implies that

$$
e^{-\int_{0}^{x+t} h_{X}(u) d u} \leq e^{-\int_{0}^{x} h_{X}(u) d u} \cdot e^{-\int_{0}^{t} h_{X}(u) d u} .
$$

That is,

$$
e^{-\int_{0}^{x} h_{X}(u+t) d u} \leq e^{-\int_{0}^{x} h_{X}(u) d u} .
$$

From the fact that $c(t)$ is increasing and (11), (9) holds, and hence the desired result follows.

Example 3 is an application of the above proposition. The following example indicates that the condition of $c(t)$ is sufficient but not a necessary one for the NBU property of $Y$.

Example 8. Assume that $X$ is a random variable having exponential distribution with mean $1 / 2$. It is clear that $X$ is NBU. Let $c(t)=(1+t) /\left(1+t^{2}\right)$ for $t \geq 0$. By some computations, we have

$$
\begin{aligned}
a(t, x)= & \int_{0}^{x}\left(c(t+u)-c(u)+h_{X}(t+u)-h_{X}(u)\right) d u \\
= & \arctan (t+x)+\frac{1}{2} \ln \left(1+(t+x)^{2}\right)-\arctan x \\
& +\frac{1}{2} \ln \left(1+x^{2}\right)+2 x t
\end{aligned}
$$

It can be verified that $a(t, x)$ is nonnegative for $t, x \geq 0$ (see also Figure 1). From (9), we conclude that $Y$ is NBU. However, it is easily obtained that $c(t)$ is increasing in $[0, \sqrt{2}-1)$ but decreasing in $(\sqrt{2}-1,+\infty)$.

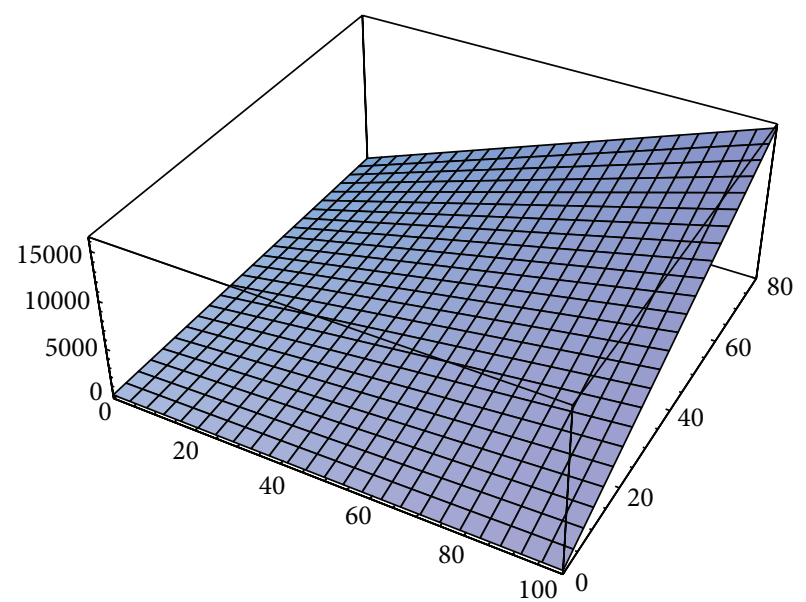

FIgURE 1: Plot of the $a(t, x)$ for $(x, t) \in[0,100] \times[0,80]$.

Proposition 9. If the random variable $X$ is NBUFR (NWUFR) and $c(t) \geq 0(\leq 0)$ for $t \geq 0$, then the random variable $Y$ is NBUFR (NWUFR).

Proposition 10. If the random variable $X$ is NBAFR $(N W A F R)$ and $\int_{0}^{t} c(u) d u \geq(\leq) t c(0)$ for $t \geq 0$, then the random variable $Y$ is NBAFR (NWAFR).

Proof. We only give the proof for the case of NBAFR. It is noted that $Y$ is NBAFR which is equivalent to that, for all $t \geq$ $0,\left(\int_{0}^{t}\left(c(u)+h_{X}(u)\right) d u\right) / t=\left(\int_{0}^{t} h_{Y}(u) d u\right) / t \geq h_{Y}(0)=c(0)+$ $h_{X}(0)$. Note that $X$ is NBAFR if and only if $\int_{0}^{t} h_{X}(u) d u / t \geq$ $h_{X}(0)$. Hence the desired result follows from the condition $\int_{0}^{t} c(u) d u \geq t c(0)$.

Remark 11. Example 3 is an application of Propositions 9 and 10. Example 6 can be regarded as a counterexample, which shows that the condition $c(t) \geq 0$ is a sufficient but not a necessary one in Propositions 9 and 10.

\section{Stochastic Comparisons of DAHR Model}

Firstly let us recall the concepts of some stochastic orders that are closely related to the main results in this section. A random variable $X$ is said to be larger than another random variable $Y$ in (a) aging intensity ordering (denoted by $X \geq_{a i} Y$ ), if

$$
\frac{h_{X}(t)}{\int_{0}^{t} h_{X}(u) d u} \leq \frac{h_{Y}(t)}{\int_{0}^{t} h_{Y}(u) d u}
$$

for all $t \geq 0$; (b) usual stochastic order (denoted by $X \leq_{s t} Y$ ) if $\bar{F}_{X}(t) \leq \bar{F}_{Y}(t)$, for all $t \geq 0$; (c) hazard rate order (denoted by $\left.X \leq_{h r} Y\right)$ if $h_{X}(t) \geq h_{Y}(t)$, for all $t \geq 0$; (d) up hazard rate order (denoted by $X \leq_{h r \uparrow} Y$ ) if $X-t \leq_{h r} Y$, for all $t \geq 0$; (e) down hazard rate order (denoted by $X \leq_{h r \downarrow} Y$ ) if $X \leq_{h r}[Y-t \mid Y>t]$, for all $t \geq 0$. For more details about stochastic orders, please refer to Shaked and Shanthikumar [21]. 


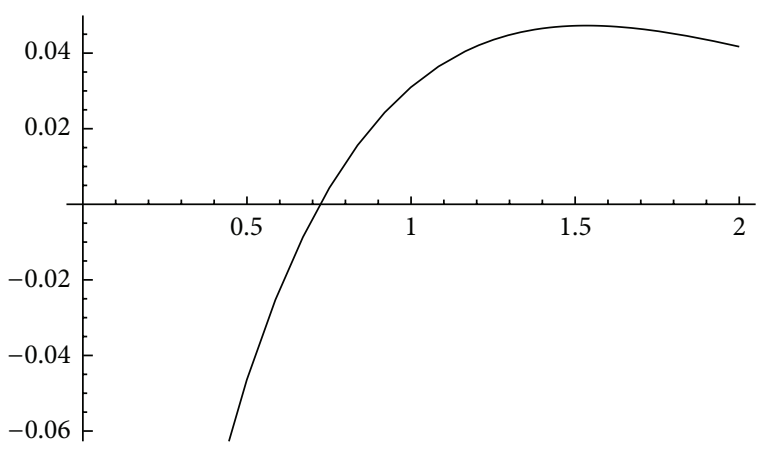

Figure 2: Plot of the $a(t)$ for $t \in[0,2]$.

In the following we give some sufficient (and necessary) conditions of stochastic ordering between random variables $X$ and $Y$. Some results are obvious and hence their proofs are omitted.

Proposition 12. Suppose $X$ and $Y$ are two nonnegative random variables satisfying (2). Then, $X \geq_{a i}\left(\leq_{a i}\right) Y$ if $c(t) / h_{X}(t)$ is increasing (decreasing) in $t \geq 0$.

Proof. Note that $X \geq_{a i} Y$ if and only if, for all $t \geq 0$,

$$
\frac{h_{X}(t)}{\int_{0}^{t} h_{X}(u) d u} \leq \frac{c(t)+h_{X}(t)}{\int_{0}^{t}\left(c(u)+h_{X}(u)\right) d u} .
$$

It is equivalent to that $\int_{0}^{t}\left(c(t) h_{X}(u)-c(u) h_{X}(t)\right) d u \geq 0$. It holds if $c(t) / h_{X}(t)$ is increasing in $t \geq 0$. The proof of the parenthetical statement is similar.

The following example indicates that the condition of the monotone property of the $c(t) / h_{X}(t)$ is sufficient but not a necessary one for the aging intensity ordering between $X$ and $Y$.

Example 13. Assume that $X$ is a random variable having exponential distribution with mean $1 / 2$. Let $c(t)=\left(1+t^{2}\right) /(1+$ $t$ ) for $t \geq 0$. By some computations, we have

$$
\begin{aligned}
a(t) & =\int_{0}^{t}\left(c(t) h_{X}(u)-c(u) h_{X}(t)\right) d u \\
& =\frac{2 t\left(1+t^{2}\right)}{1+t}-t^{2}+2 t+4 \ln (1+t) .
\end{aligned}
$$

It can be verified that $a^{\prime}(t) \geq 0$ for $t \geq 0$, and hence $a(t)$ is increasing in $t \geq 0$ (see also Figure 2). Note that $a(0)=0$. Thus $a(t) \geq 0$, for all $t \geq 0$, and hence $X \geq_{a i} Y$. However, it is easily obtained that $c(t) / h_{X}(t)$ is decreasing in $[0, \sqrt{2}-1)$ but increasing in $(\sqrt{2}-1,+\infty)$.

Proposition 14. Suppose $X$ and $Y$ are two nonnegative random variables satisfying (2). Then, $X \geq_{s t}\left(\leq_{s t}\right) Y$ if and only if $\int_{0}^{t} c(u) d u \geq(\leq) 0$, for all $t \geq 0$.

The following corollary follows immediately from the proposition above.
Corollary 15. If $c(t) \geq(\leq) 0$, for all $t \geq 0$, then $X \geq_{s t}\left(\leq_{s t}\right) Y$.

Proposition 16. Suppose $X$ and $Y$ are two nonnegative random variables satisfying (2). Then, $X \geq_{h r}\left(\leq_{h r}\right) Y$ if and only if $c(t) \geq(\leq) 0$, for all $t \geq 0$.

Proposition 17. Suppose that $X$ and $Y$ are two nonnegative random variables satisfying (2). Then, $X \leq_{h r \uparrow}\left(\geq_{h r \uparrow}\right) Y$ if and only if $h_{X}(y+t)-h_{X}(t)-c(t) \geq(\leq) 0$, for all $y \geq 0$ and $t \geq 0$.

Proof. Note that $X \leq_{h r \uparrow} Y$ if and only if

$$
\frac{\exp \left[-\int_{0}^{x} h_{Y}(u) d u\right]}{\exp \left[-\int_{0}^{x+t} h_{X}(u) d u\right]}
$$

is increasing in $x$, for all $t \geq 0$. It is equivalent to the fact that

$$
\exp \left[\int_{0}^{x+t} h_{X}(u)-\int_{0}^{x} h_{Y}(u) d u\right]
$$

is increasing in $x$, which is equivalent to the fact that its derivative is nonnegative; that is, $h_{X}(y+t)-h_{X}(t)-c(t) \geq 0$, for all $y \geq 0$ and $t \geq 0$. It follows from the condition. The proof of the parenthetical statement is similar.

Proposition 18. Suppose that $X$ and $Y$ are two nonnegative continuous random variables satisfying (2). Then, $X \leq_{h r \downarrow}\left(\geq_{h r \downarrow}\right) Y$ if and only if $h_{X}(y)-h_{X}(t+y)-c(t+y) \geq(\leq) 0$, for all $y \geq 0$ and $t \geq 0$.

Its proof is similar to that of Proposition 17 and hence is omitted.

\section{Conflict of Interests}

The authors declare that there is no conflict of interests regarding the publication of this paper.

\section{Acknowledgment}

The research was supported by the National Natural Science Foundation of China (71361020).

\section{References}

[1] D. R. Cox, "Regression models and life-tables," Journal of the Royal Statistical Society, vol. 34, pp. 187-220, 1972.

[2] P. L. Gupta and R. C. Gupta, "Ageing characteristics of the Weibull mixtures," Probability in the Engineering and Informational Sciences, vol. 10, no. 4, pp. 591-600, 1996.

[3] D. R. Cox and D. Oakes, Analysis of Survival Data, Chapman \& Hall, New York, NY, USA, 1984.

[4] D. Kumar and U. Westberg, "Maintenance scheduling under age replacement policy using proportional hazards model and TTT-plotting," European Journal of Operational Research, vol. 99, no. 3, pp. 507-515, 1997.

[5] J. Dupuy, "The proportional hazards model with covariate measurement error," Journal of Statistical Planning and Inference, vol. 135, no. 2, pp. 260-275, 2005. 
[6] J. W. Lau, "Bayesian semi-parametric modeling for mixed proportional hazard models with right censoring," Statistics \& Probability Letters, vol. 76, no. 7, pp. 719-728, 2006.

[7] X. Zhao and X. Zhou, "Proportional hazards models for survival data with long-term survivors," Statistics \& Probability Letters, vol. 76, no. 15, pp. 1685-1693, 2006.

[8] R. Li and L. Chambless, "Test for additive interaction in proportional hazards models," Annals of Epidemiology, vol. 17, no. 3, pp. 227-236, 2007.

[9] Q. Yu, "A note on the proportional hazards model with discontinuous data," Statistics \& Probability Letters, vol. 77, no. 7, pp. 735-739, 2007.

[10] R. C. Gupta and R. D. Gupta, "Proportional reversed hazard rate model and its applications," Journal of Statistical Planning and Inference, vol. 137, no. 11, pp. 3525-3536, 2007.

[11] R. C. Gupta and H. Wu, "Analyzing survival data by proportional reversed hazard model," International Journal of Reliability and Applications, vol. 2, pp. 1-26, 2001.

[12] X. Li and Z. Li, "A mixture model of proportional reversed hazard rate," Communications in Statistics: Theory and Methods, vol. 37, no. 18-20, pp. 2953-2963, 2008.

[13] A. K. Nanda and S. Das, "Dynamic proportional hazard rate and reversed hazard rate models," Journal of Statistical Planning and Inference, vol. 141, no. 6, pp. 2108-2119, 2011.

[14] F. J. Aranda-Ordaz, "An extension of the proportional-hazards model for grouped data," Biometrics, vol. 39, no. 1, pp. 109-117, 1983.

[15] D. C. Thomas, "Use of auxiliary information in fitting nonproportional hazards models," in Modern Statistical Methods in Chronic Disease Epidemiolgy, E. D. S. Moolgavkar and R. L. Prentice, Eds., pp. 197-210, Wiley, New York, NY, USA, 1986.

[16] N. E. Breslow and N. E. Day, Statistical Methods in Cancer Research-vol 1, The Design and Analysis of Case-Control Studies, IARC, Lyon, France, 1987.

[17] M. S. Finkelstein and V. Esaulova, "On an inverse problem in mixture failure rates modelling," Applied Stochastic Models in Business and Industry, vol. 17, no. 2, pp. 221-229, 2001.

[18] H. J. Lim and X. Zhang, "Semi-parametric additive risk models: application to injury duration study," Accident Analysis and Prevention, vol. 41, no. 2, pp. 211-216, 2009.

[19] R. E. Barlow and F. Proschan, Statistical Theory of Reliability and Life Testing, Holt, Rinehart and Winston, New York, NY, USA, 1981.

[20] A. Müller and D. Styan, Comparison Methods for Stochastic Models and Risks, John Wlley \& Sons, Westsusse, UK, 2002.

[21] M. Shaked and J. G. Shanthikumar, Stochastic Orders and Their Applications, Academic Press, San Diego, Calif, USA, 1994. 


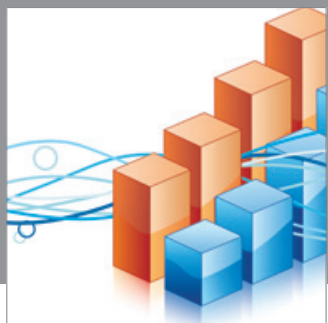

Advances in

Operations Research

mansans

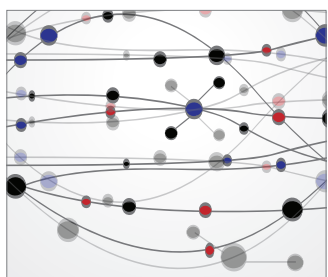

The Scientific World Journal
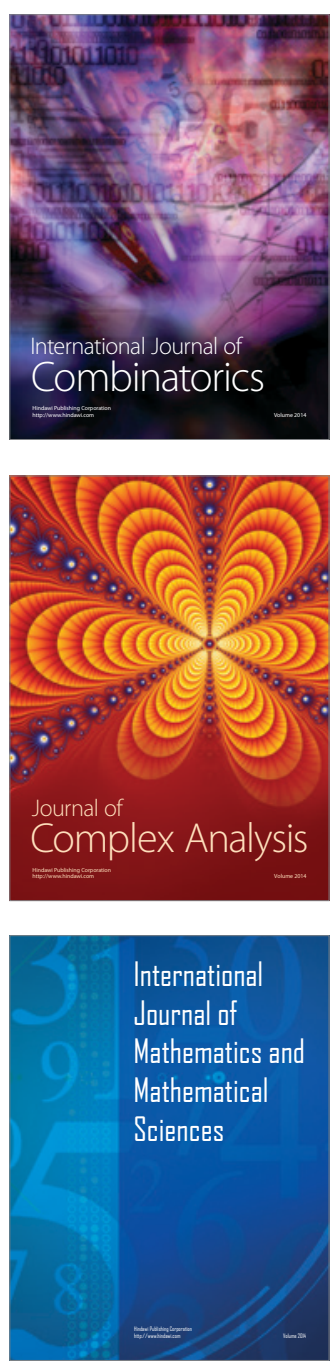
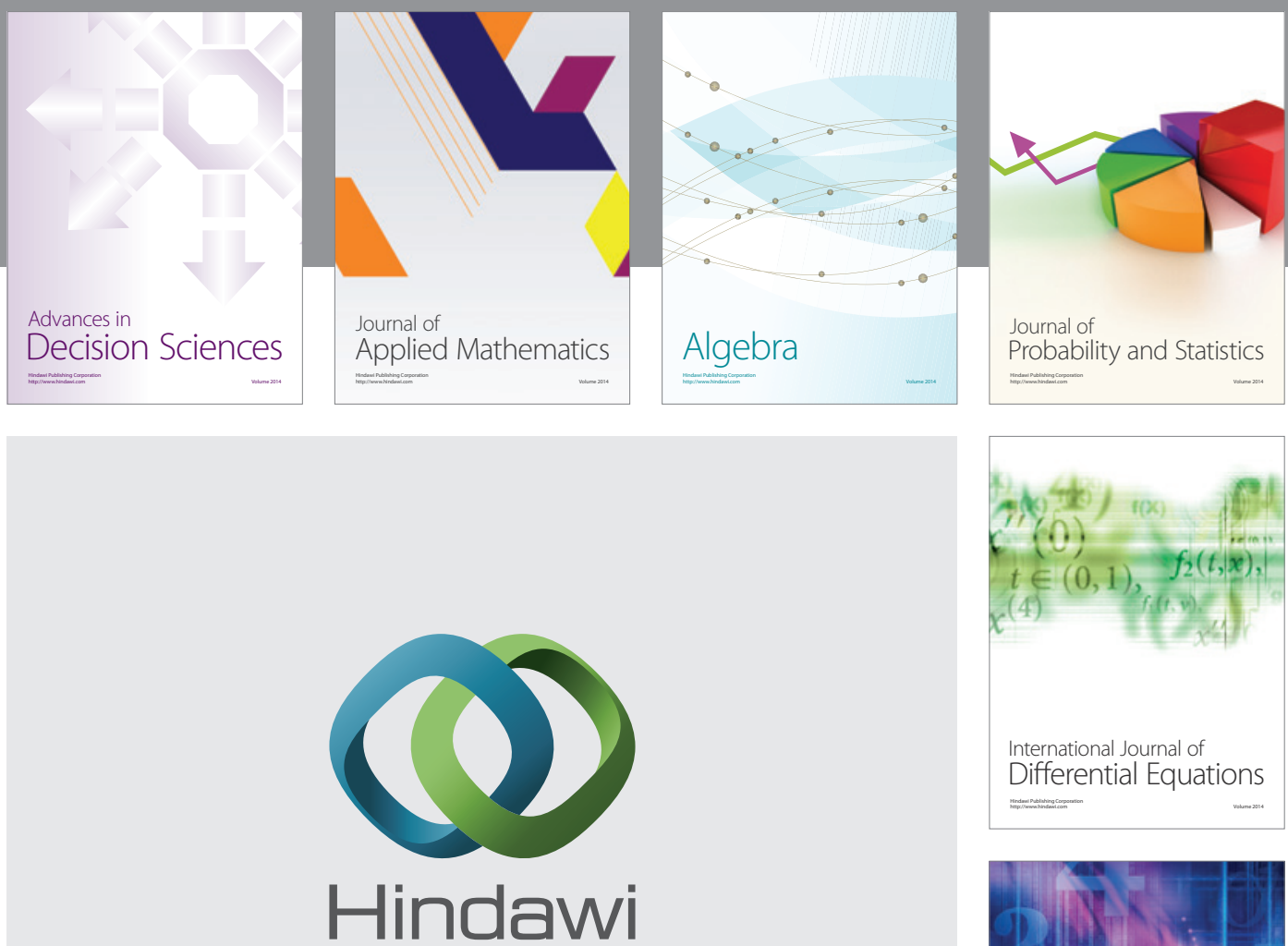

Submit your manuscripts at http://www.hindawi.com
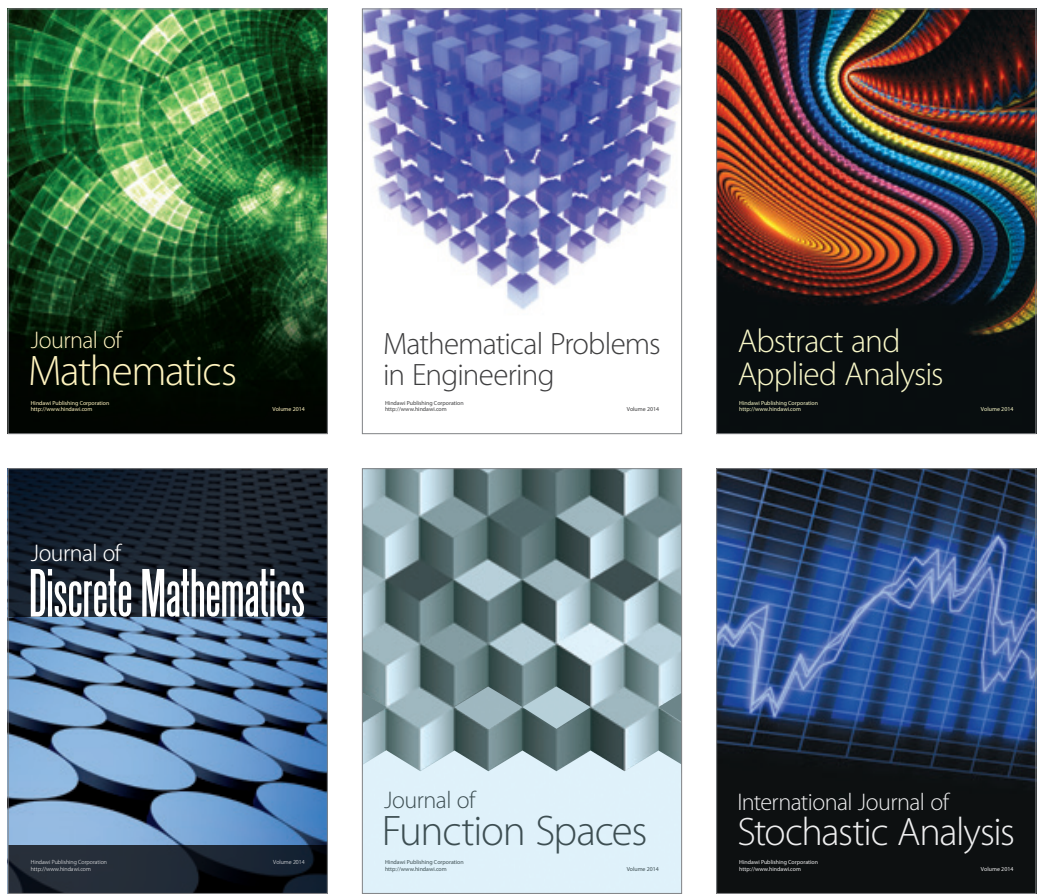

Journal of

Function Spaces

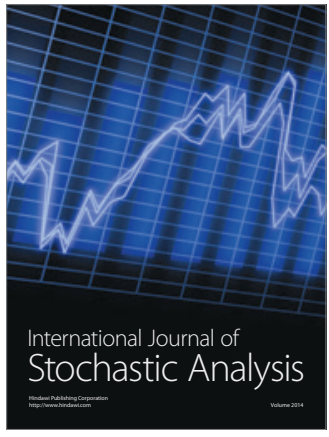

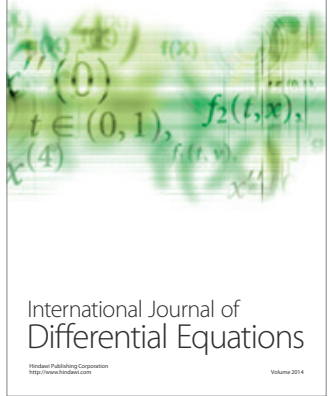
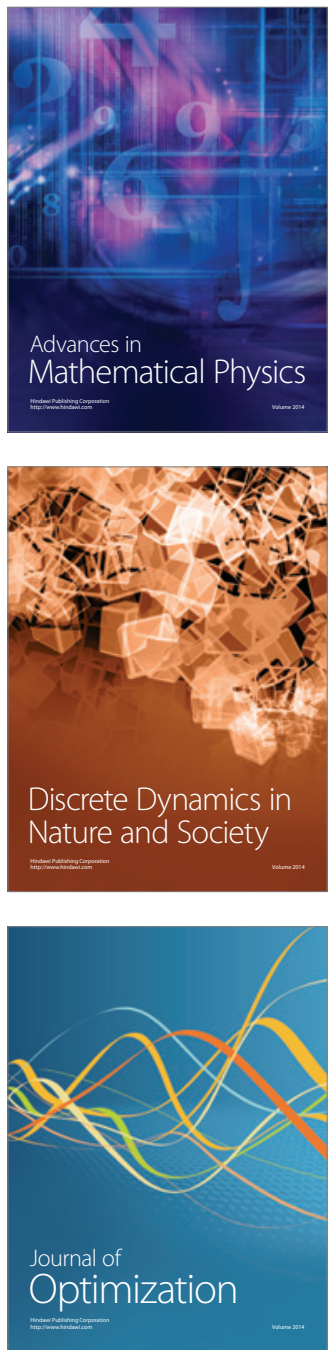